\title{
Violation of fluctuation-dissipation relations for electron transfer in nonpolar solvents
}

\author{
Gerdenis Kodis $\odot$, Ian R. Gould, and Dmitry V. Matyushov $\odot^{*}$ \\ Department of Physics and School of Molecular Sciences, Arizona State University, P.O. Box 871504, Tempe, Arizona 85287-1504, USA
}

(Received 13 November 2020; accepted 12 January 2021; published 4 February 2021)

\begin{abstract}
Nonpolar materials are increasingly employed as media for electron transfer, particularly in applications related to solar energy conversion. What should be the mechanism of activation for electron tunneling in the absence of rotating permanent dipoles considered in standard theories is not clear. We suggest that compression and decompression (density) fluctuations shifting positions of polarizable molecules of the medium is the mechanism for radiationless transitions. These fluctuations affect the induction interactions between the medium induced dipoles and the localized electron (induction forces). Solvent reorganization energy must be a signature of such fluctuations, but it has never been directly measured for electron transfer in nonpolar media. Here, absorption and emission spectra of a charge-transfer complex are analyzed as functions of temperature in cyclohexane. Significant reorganization energies, $0.2-0.5 \mathrm{eV}$, are found. They strongly differ between the ground and photoexcited charge-transfer states in violation of fluctuation-dissipation relations establishing the basis for modern theories of electron transfer. The reorganization energies are decaying functions of temperature, also in violation of the macroscopic fluctuation-dissipation relations.
\end{abstract}

DOI: 10.1103/PhysRevResearch.3.013109

\section{INTRODUCTION}

Transfer of electrons between molecules is a process fundamental to the hopping limit of electron conductivity [1,2], redox chemistry [3], and biology [4]. It is a major step in energy chains of mitochondria and in natural photosynthesis $[4,5]$. All energy produced by biology comes, through electron and proton transfer, from the transformation of the chemical potential of high-energy electronic states into other forms of energy. Mechanistic issues related to activated hops of electrons between localized states within the molecules are still explored, in particular in application to the transport of electrons in complex biological systems [6]. At the same time, the demand for affordable sources of energy drives the research effort to designing efficient artificial photosynthetic systems [7-9]. They often employ media very different from aqueous solutions in which energy complexes of biology operate and conventional polar solvents used in classical research of electron-transfer reactions $[10,11]$. In particular, the use of nonpolar materials/solvents for molecular charge transfer [12-15] and charge transport [16] has become increasingly widespread since these media avoid substantial solvation energies of polar solvents trapping electrons at their hopping sites [17] and thus increase efficiency of solar cells [18].

The theory of electron transfer in nonpolar solvents involves significant fundamental distinctions from the tradi-

\footnotetext{
*dmitrym@asu.edu

Published by the American Physical Society under the terms of the Creative Commons Attribution 4.0 International license. Further distribution of this work must maintain attribution to the author(s) and the published article's title, journal citation, and DOI.
}

tional theory adopted for polar liquid solvents [19]. Both the driving forces promoting electron transfer (induction interactions versus electron-dipole interactions) and thermodynamic properties of medium fluctuations affecting electron transfer are different in nonpolar solvents. Spectroscopy of chargetransfer optical bands has traditionally played a decisive role in testing theories of electron transfer [20-22]. Here, spectroscopy of charge-transfer bands in a nonpolar liquid solvent is used to elucidate the fundamental differences between electron transfer in polar and nonpolar solvents. We demonstrate a dramatic violation of fluctuation-dissipation relations establishing the foundation of present-day theories of electron transfer.

Theoretical basis for describing electron transfer between molecules in polar solvents was established by the Marcus theory [10]. This is a generic Gaussian model describing the modulation of electronic energies in molecules by thermally induced rotations of permanent dipoles in the liquid. It states that overcoming a gap in energy between two localized states of the electron is required for electron tunneling and transport. If the equilibrium gap between the donor and acceptor electronic states is $\langle\Delta E\rangle$, the probability of the reaction is the product of the tunneling probability and the Gaussian probability of reaching the resonance

$$
k_{\mathrm{ET}} \propto V^{2} e^{-\langle\Delta E\rangle^{2} /\left(2 \sigma^{2}\right)} .
$$

Here, $V$ in front of the Gaussian probability term is the electron-transfer matrix element which quantifies the tunneling probability $[23,24]$.

The rate constant in Eq. (1) is brought to the Arrhenius form by applying the static limit of the fluctuation-dissipation theorem (FDT) [25] to the Gaussian width of the energy gap $\sigma^{2}=\left\langle(\delta \Delta E)^{2}\right\rangle, \delta \Delta E=\Delta E-\langle\Delta E\rangle$. This fluctuation relation states that the variance of equilibrium thermal 
fluctuations of a macroscopic variable scales linearly with temperature (Johnson-Nyquist noise [26]).

$$
\text { Rule I : } \quad \sigma^{2}=2 \lambda k_{\mathrm{B}} T \propto T .
$$

When this temperature scaling is used in Eq. (1), one arrives at the Arrhenius law for the rate constant $k_{\mathrm{ET}}$.

The proportionality constant between the Gaussian variance and temperature in Eq. (2) is the Marcus reorganization energy $\lambda$ [27]. It enters yet another fluctuation-dissipation relation connecting the linear response of a system to an external perturbation with thermally induced fluctuations around equilibrium. This result goes back to the Onsager regression theorem [28] stating that small fluctuations out of equilibrium can be viewed as regression from nonequilibrium states externally driven by small perturbations. In the modern formulation of the linear response theory [25,29], if the variable $A$ is driven out of equilibrium by the force $f$ conjugate to $A$, the susceptibility $\chi=\Delta A / f$ can be expressed as the variance of the same variable $A$ scaled with the inverse temperature: $\chi=\beta\left\langle(\delta A)^{2}\right\rangle, \beta=\left(k_{\mathrm{B}} T\right)^{-1}$.

When applied to the theory of electron-transfer reactions [20,27], the fluctuation-dissipation relation establishes the connection between the equilibrium energy gaps for the forward, $\langle\Delta E\rangle_{1}$, and backward, $\langle\Delta E\rangle_{2}$, transitions with the Marcus reorganization energy.

$$
\text { Rule II : } \lambda=\frac{1}{2}\left|\langle\Delta E\rangle_{1}-\langle\Delta E\rangle_{2}\right| .
$$

When transfer of the electron is caused by light in optical charge-transfer transitions, $\langle\Delta E\rangle_{i}$ are associated with the maxima of the absorption and emission bands. Equation (3) then establishes the connection between the reorganization energy and the Stokes shift of the charge-transfer optical band [20].

The requirement of linear response of the medium to electron transfer places one more constraint on the parameters involved. This is the requirement of equal Gaussian variances $\sigma^{2}$ for the forward and backward electronic transitions. If one applies $\sigma_{1}^{2}$ to the rate of the forward transition $1 \rightarrow 2$ and $\sigma_{2}^{2}$ to the backward transition $2 \rightarrow 1$, the requirement of using linear response theories is the equality between them,

$$
\text { Rule III : } \sigma_{1}^{2}=\sigma_{2}^{2} \text {. }
$$

For optical transitions, $\sigma_{i}$ define inhomogeneous broadening of optical lines. Rule III then implies that the absorption and emission lines should carry equal widths.

Rules I-III specify the fluctuation-dissipation relations in application to electron transfer. They are numbered by their fundamental significance. Rule I is by far most significant for applications since it brings the electron-transfer kinetics into the realm of the Arrhenius law. Rule II leads to a practical recipe [10] connecting the average energy gap for electronic transitions with the reaction free energy $\Delta G$ of thermally activated electron transfer

$$
\langle\Delta E\rangle_{i}=\Delta G \pm \lambda,
$$

where " + " is used for the forward reaction $1 \rightarrow 2(i=1)$ and "-" applies to the backward reaction $2 \rightarrow 1(i=2)$. The reaction free energy for thermal electron transfer is given by the mean of two average vertical excitation energies

$$
\Delta G=\frac{1}{2}\left(\langle\Delta E\rangle_{1}+\langle\Delta E\rangle_{2}\right) .
$$

Finally, rule III allows one to express the activation barriers for the forward and backward reactions in terms of only two parameters, $\lambda$ and $\Delta G$. The forward and backward rates automatically satisfy the detailed balance.

All three rules in Eqs. (2)-(4) are derived from the same analytical framework of linear-response theories. It is therefore clear that they all should be violated when linear response is compromised [27]. Before turning to this point, it is first important to establish which systems and reactions are most likely to follow such rules.

The Marcus theory of electron transfer has seen its greatest success in application to reactions in polar liquid solvents where it has been most extensively tested [11,23]. An important physical signature of electron transfer in polar liquids is the dominance of long-range interactions between the transferred electron and polar (dipolar) molecules of the solvent. The number of solvent molecules involved in such interactions is large, which makes the electron-medium interaction energy a quasimacroscopic stochastic (fluctuating) variable. The macroscopic limit is where the fluctuation-dissipation relations are expected to hold exactly. The long range of the electrostatic interactions with the polar medium is what ensures success of the Marcus theory from the fundamental standpoint.

Even though the Marcus theory has been universally successful for reactions in polar media, the validity of rules I-III has not been uniformly tested. Most reactions follow the Arrhenius law and rule I can be viewed as well supported. Nevertheless, curved [30-32] and bell-shaped [33,34] forms of the dependence of the reaction rate on the inverse temperature have been reported. They received theoretical support in terms of the dependence of $\lambda$ on $T$ [34]. Equation (2) holds as a formal definition of the reorganization energy, but one often has to account for its temperature dependence, which makes the actual temperature variation of the reaction rate non-Arrhenius. Rule II leads to Eq. (5), which has been supported by numerous observations [35-42] of the bell-shaped energy gap law, i.e., an inverted parabola predicted by Marcus for the rate plotted versus the reaction free energy $\Delta G$ [10]. Finally, experimental testing of the validity of rule III is a challenging problem and there are only a few examples of charge-transfer spectra when deviations from this prescription can be separated from other complications [43-45]. More detailed evidence of violation of rule III comes from theoretical [27,46,47] and simulation [48-50] studies. Such violations of rule III are detected either as asymmetry in the energy-gap laws between charge separation and charge recombination reactions [51] (see Ref. [15] for a recent review) or as band asymmetry between absorption and emission charge-transfer optical lines $[43,44,52]$. The origin of these effects is often difficult to pinpoint specifically since they can originate either from changes in frequencies of intramolecular vibrations [53-55] (such as the Duschinsky rotation [56]) or from other not well characterized effects such as the molecular polarizability [27] and, to a lesser extent, from nonlinear solvation $[57,58]$. Nonlinear solvation is, however, very significant for half redox reactions of simple ions [48,50]. 
A significant class of electron-transfer reactions where the fluctuation-dissipation rules are expected to break is electron transfer in nonpolar media [59,60]. Nonpolar media are defined as those where both the dipole moment of the solvent is zero and the quadrupole moment does not produce a significant reorganization energy. Molecular quadrupoles affect the solute-solvent electrostatics through molecular orientations, similarly to the dipoles [61-64], although the component of the reorganization energy arising from molecular translations is more prevalent for quadrupoles than for dipoles [65,66]. Nonpolar media present a limiting case for which the standard models [10] offer no physical mechanism for electron transfer since there are no permanent dipoles or quadrupoles to allow nonequilibrium electrostatic fluctuations to overcome the activation barrier. One needs a nuclear fluctuation shifting energies of electronic states. Physical interactions allowing such fluctuations are also electrostatic in character. However, instead of interactions of the transferred electron with the permanent dipoles of the medium, the interaction is with induced dipoles, via the induction forces. The nuclear fluctuations are afforded by translations of the induced dipole, i.e., through density fluctuations. To study this novel mechanism, two questions are addressed here: (1) How significant is the solvent reorganization energy that can be gained through fluctuations of induction interactions? and (2) What are the observable consequences of a new mechanism of promoting electron transfer?

The induction interactions decay much faster with the distance than the electron-dipole interactions and fewer molecules of the medium are involved. This basic physics makes the solute-solvent interaction potential a microscopic variable, instead of a quasimacroscopic variable for polar interactions, thus invalidating the fluctuation-dissipation relations strictly applicable in the macroscopic limit [19]. Here, we support these views by reporting absorption and emission charge-transfer bands in nonpolar cyclohexane at a number of temperatures. The mechanism of electron transfer activated by induction forces is validated.

\section{RESULTS AND DISCUSSION}

Intramolecular charge transfer can be often viewed from the electrostatic standpoint as the alteration of the solute dipole moment from $\mathbf{m}_{1}$ in the initial state to $\mathbf{m}_{2}$ in the final state; $\Delta \mathbf{m}=\mathbf{m}_{2}-\mathbf{m}_{1}$ is the change in the molecular dipole. The induction interaction between the solute and the polarizable solvent is the free energy of creating induced dipoles at the molecules in the liquid carrying the molecular polarizability $\alpha$

$$
F_{e i}=-m_{i}^{2} U, \quad U=\sum_{j} v(j),
$$

where

$$
v(j)=\left(\alpha / r_{j}^{6}\right)\left[1+P_{2}\left(\hat{\mathbf{m}} \cdot \hat{\mathbf{r}}_{j}\right)\right]
$$

and $P_{2}(x)$ is the second-order Legendre polynomial. It is given as a function of the dot product between the unit vectors $\hat{\mathbf{m}}$ of the solute dipole and $\hat{\mathbf{r}}_{j}$ pointing to the molecule $j$ in the solvent. The sum in Eq. (7) runs over all $N$ molecules of the solvent and the solute orientations are averaged in calculating
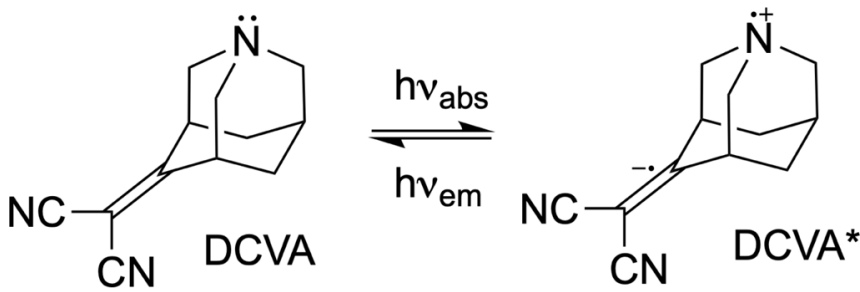

FIG. 1. Charge transfer in DCVA molecule.

the statistical averages. This is the reason why $\hat{\mathbf{m}}$ carries no dependence on the charge-transfer state $(i=1,2)$.

The instantaneous transition energy is shifted by $\Delta F_{e}=$ $F_{e 2}-F_{e 1}$ and the average interaction energy in each chargetransfer state $\left\langle\Delta F_{e}\right\rangle_{i}$ contributes to the solvent-induced spectroscopic shift of the transition band $[67,68]$. The difference of equilibrium solvent-induced shifts is the solvent-induced Stokes shift

$$
\left\langle\Delta F_{e}\right\rangle_{1}-\left\langle\Delta F_{e}\right\rangle_{2}=h v_{s}^{\mathrm{St}}=2 \lambda .
$$

As in the standard Marcus formulation [Eq. (2)], the reorganization energy arises from the Gaussian variance of the solute-solvent interaction energy [19]

$$
\lambda=\frac{1}{2} \beta\left(\delta m^{2}\right)^{2}\left\langle(\delta U)^{2}\right\rangle,
$$

where $\delta m^{2}=m_{2}^{2}-m_{1}^{2}$.

The reorganization energy due to induction interactions can be calculated by using perturbation formalisms of the liquid state theory [69]. The resulting expression, derived in more detail in Appendix A [Eq. (A11)], scales linearly with the squared molecular polarizability of the solvent $\alpha$. This dependence can be cast, by applying the Clausius-Mossotti equation, in terms of the squared refractive index $\epsilon_{\infty}=n_{D}^{2}$ and the packing fraction of the solvent $\eta=(\pi / 6) \rho \sigma_{s}^{3}$. The latter defines the fraction of the entire volume $\Omega$ occupied by $N$ liquid molecules with the diameter $\sigma_{s} ; \rho=N / \Omega$ is the number density of the solvent. Here, we convert the general perturbation theory to practical expressions applicable to analyzing spectral shifts in nonpolar solvents.

In many practical situations involving charge-transfer bands, the dipole moment of the ground state is much lower than the dipole moment of the charge-transfer state. This is the situation with the dicyanovinylazaadamantane (DCVA) molecule [70,71] studied here (Fig. 1). This molecule presents a clean charge-transfer complex in which both absorption and emission charge-transfer transitions can be observed with a negligible mixing from locally excited states. It shows little solvatochromism of its absorption band suggesting that $m_{1}$ is small compared to $\Delta m$. The energy of the absorption band $h v^{\text {abs }}=\langle\Delta E\rangle_{1}$, shifted from the vacuum transition energy $h v_{0}^{\text {abs }}$ by the induction stabilization, becomes [Eqs. (A3) and (A6)]

$$
h v^{\mathrm{abs}}=h v_{0}^{\mathrm{abs}}+\Delta F_{e}\left(a_{1}\right),
$$

where

$$
\Delta F_{e}\left(a_{i}\right)=-\frac{(\Delta m)^{2}}{a_{i}^{3}} \frac{\epsilon_{\infty}-1}{\epsilon_{\infty}+2}
$$


Here, $a_{i}$ is the effective radius of the solute for the absorption $(i=1)$ and emission $(i=2)$ transitions. The dipole moments enter the induction shift as $m_{2}^{2}-m_{1}^{2}$ and the error of neglecting $m_{1}$ is of the order $m_{1}^{2} / m_{2}^{2}$. It is offset in practical calculations by adjusting the effective radii $a_{i}$.

The timescale of emission in most cases far exceeds the relaxation time of the solvent density around the donor-acceptor complex. The new local structure necessitates a different effective radius of the solute $a_{2}$. The energy of the emission line $h v^{\mathrm{em}}=\langle\Delta E\rangle_{2}$ is found from the perturbation expansion and can be written as follows [Eq. (A4)]:

$$
h v^{\mathrm{em}}=h v_{0}^{\mathrm{em}}+\Delta F_{e}\left(a_{2}\right)-2 \lambda\left(a_{2}\right) .
$$

The average induction energy $\Delta F_{e}\left(a_{i}\right)$ in Eq. (12) scales linearly with $\alpha(\Delta m)^{2}$, while the reorganization energy, which arises from the second cumulant of the interaction energy [Eq. (10)], scales quadratically with this factor. This scaling allows one to write $\lambda$ in terms of $\Delta F_{e}\left(a_{2}\right)$

$$
\lambda\left(a_{2}\right)=\beta\left(\Delta F_{e}\left(a_{2}\right)\right)^{2} f\left(\eta, a_{2} / \sigma_{s}\right),
$$

where $f\left(\eta, a_{2} / \sigma_{s}\right)$ [Eq. (A14)] is numerically calculated in Appendix A with the use of the Percus-Yevick (PY) solution for the density structure factor $S(k)$ for the fluid of hard spheres.

Only the dependence of the reorganization energy on temperature, $\lambda \propto \beta, \beta=\left(k_{\mathrm{B}} T\right)^{-1}$ in Eq. (14), is essential for the band shape analysis. The proportionality of $\lambda$ to $\beta$ is a nontrivial result. This dependence is canceled out by the value $S(0) \propto T$ for solvation in polar solvents dominated by long-range Coulomb interactions, which translate to small $k$-values in reciprocal space when integration is performed. This does not happen for much more short-ranged induction interactions and $\lambda \propto \beta$ is preserved (Fig. 6). Further, the gas-phase energies $h v_{0}^{\text {abs/em }}$ in Eqs. (11) and (13) are equal if there is no classical intramolecular reorganization energy of the solute, but can differ to reflect the Stokes shift from internal classical vibrations. The solvent-induced Stokes shift is therefore defined as

$$
h \Delta v_{s}^{\mathrm{St}}=h\left(v^{\mathrm{abs}}-v^{\mathrm{em}}\right)-h\left(v_{0}^{\mathrm{abs}}-v_{0}^{\mathrm{em}}\right) .
$$

Equations (11) and (13) are consistent with the result of the linear (Gaussian) model [Eq. (9)] at $a=a_{1}=a_{2}$. In this case, the term $\Delta F_{e}(a)$ cancels in the difference of absorption and emission energies, leaving the solvent-induced Stokes shift equal to $2 \lambda$ (rule II). If $a_{1} \neq a_{2}$, the reorganization energy in each state can be given as

$$
\lambda_{i} \propto \beta\left(\Delta F_{e}\left(a_{i}\right)\right)^{2} .
$$

The term $\left(\Delta F_{e}\left(a_{i}\right)\right)^{2}$ [Eq. (12)] absorbs the dependence of $\lambda_{i}$ on the solute dipole $\Delta m$, on the solvent refractive index, and on the effective solute size $a_{i}$.

The important distinction of solvent reorganization by induction interactions compared to the standard results in polar solvents [10] is stressed by $\lambda_{i} \propto \beta$ in Eq. (16). This result makes the Gaussian width in Eq. (1) independent of temperature at constant density, in violation of rule I. A small residual temperature dependence of the Gaussian width is still preserved through solvent's expansion when experiments are done at constant pressure. Rule I is violated because of the short spatial range of the solute-solvent induction interactions.
Fluctuations of the interaction energy are driven by local repacking of the molecular repulsive cores. The probability of such fluctuations is determined by an entropic penalty, which alters the temperature dependence of the corresponding variance. In contrast, fluctuations of permanent dipoles in polar liquids are mostly driven by the enthalpy, even though translations of permanent dipoles (density fluctuations) are still mostly responsible for the entropic component of the activation barrier [34].

Equation (14) is derived for a spherical solute with the point dipole moment at the center, which changes its magnitude through charge transfer. Assuming that the solute radius $a$ is not altered by charge transfer, the formalism presented in Eqs. (7)-(12) leads to the violation of rule I while preserving rules II and III. However, our experimental results show a clear violation of all three rules. The reason is a strong dependence of all averages involved on the solute radius $a$. The reorganization energy scales as $a^{-9}$ in Eq. (14) (Fig. 5). Therefore, changing the solute radius, through solvent's contraction, strongly affects the resulting inhomogeneous width in the band shape analysis.

The solute radius affecting both the induction-induced spectral shift $\Delta F_{e}(a)$ [Eq. (12)] and the reorganization energy [Eq. (14)] is an effective radius. It is specified in quantitative solvation theories to yield the average interaction energy, scaling with the distance as $r^{-n}$, in terms of the effective interaction radius [19]

$$
\frac{1}{a^{n-3}}=(n-3) \int_{0}^{\infty} \frac{d r}{r^{n-2}} g_{0 s}(r),
$$

where $g_{0 s}(r)$ is the solute-solvent radial distribution function. The sensitivity of such an effective radius to the local structure grows with increasing power $n$. For instance, the term $1 / a_{i}^{3}$ in Eq. (12) is obtained by applying $n=6$ in Eq. (17), which corresponds to $r^{-6}$ scaling of the interaction of the solute dipole with the induced solvent dipole in Eq. (8). However, the scaling with the distance changes for the reorganization energy. When the induction interaction energy is used to calculate the variance in Eq. (10), one has to take the statistical average of the squared potential energy scaling as $r^{-12}, n=$ 12. This is the reason for the scaling $\lambda \propto a^{-9}$ for the induction reorganization energy.

The need to specify an effective solute size does not usually create significant conceptual problems since this parameter is typically chosen to fit observations. However, the sensitivity of short-range interactions to the local structure, and the corresponding sensitivity of $\lambda$ to the effective radius, produce a new physical reality since the local structure of the solvent is altered by charge transfer. This alteration, which typically amounts to the compression of the solvation shell upon increasing the charge magnitude $[48,72]$, causes changes in the effective solute size [73] as described by the continuum viscoelastic model of solvation $[60,74]$. That model addresses the appearance of fast, subpicosecond dynamics caused by changing solute size [75], but does not clarify the physical origin of interactions responsible for the Stokes shift and the reorganization energy. It is this latter problem that is addressed here. Contraction of the solute size upon changing charge is reported by simulations even in polar solvents $[48,72]$. Nonpolar solvents are typically more compressible, due to 


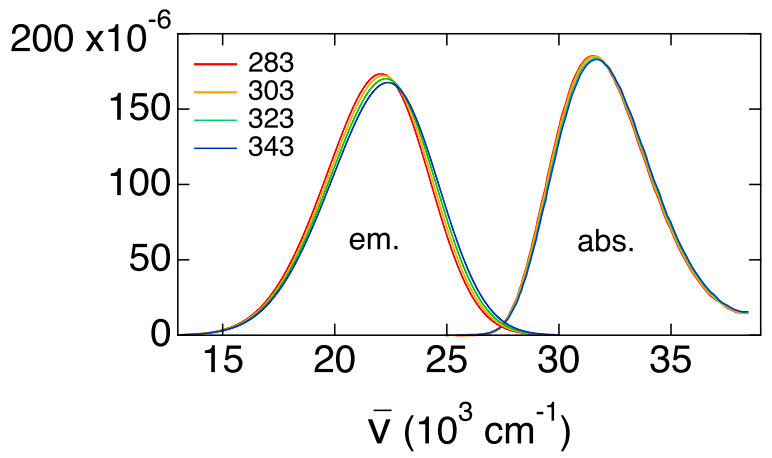

FIG. 2. Normalized absorption (abs.) and emission (em.) spectra of DCVA in cyclohexane at the temperatures listed in the plot.

generally lower cohesive energy, and the compression effect must be greater than in polar solvents. A change in the effective solute size leads to a corresponding change in $\lambda$ not anticipated by linear solvation theories. This is the picture found in this study from the analysis of spectroscopic data for DCVA recorded in cyclohexane in the temperature range 280-360 K (Figs. 2 and 3).

Ground state absorption spectra were measured with the Varian Cary 50 UV-Vis Spectrophotometer (Agilent). Steady state fluorescence spectra were measured using a Photon Technology International MP-1 Spectrometer and corrected for the detection system response. Excitation was provided by a $75 \mathrm{~W}$ xenon arc lamp and single-grating monochromator. Fluorescence was detected at $90^{\circ}$ to the excitation beam via a single-grating monochromator and an R928P photomultiplier tube operating in the single-photon-counting mode. A $1 \mathrm{~cm}$ quartz screw-cap cuvette (Spectrocell) was placed into a Quantum Northwest Peltier-Based Temperature-Controlled Cuvette Holder TLC 50 connected to the TC 125 Temperature Controller. Temperature was calibrated with an alcohol glass thermometer.

The band shape analysis of experimental data follows the standard protocol in which the spectral band is given by a vibronic progression of individual excitations inhomogeneously broadened by the solvent [22,43,76-78] (Appendix B).

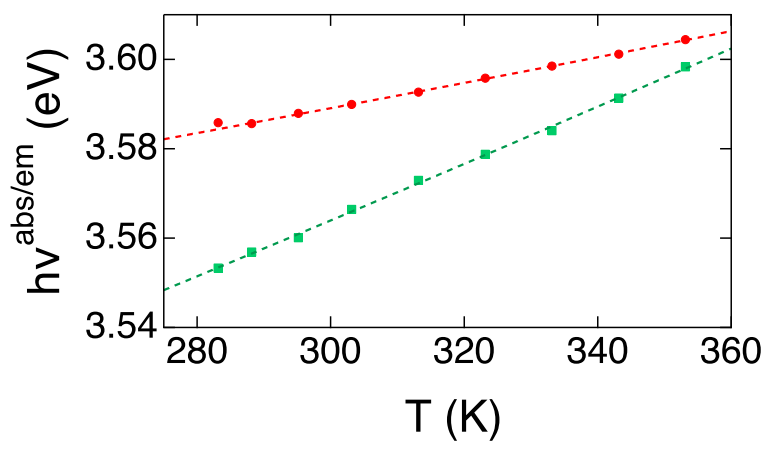

FIG. 3. Absorption (red) and emission (green) transition energies [Eq. (22)] vs $T$. The results for emission are shifted upward by $0.5 \mathrm{eV}$ to bring the two lines to the same scale. The dashed lines are fits of the experimental data (points) to Eqs. (11)-(13) in which the solute radius $a_{i}$ is adopted as the fitting parameter. The straight dashed lines shown in the figure require $a_{1}=5.08 \AA$ and $a_{2}=3.92 \AA$.
The Franck-Condon spectral density is given as a Poisson distribution weighted sum of Gaussian functions

$$
\mathrm{FC}(v) \propto e^{-S} \sum_{n=0}^{\infty} \frac{S^{n}}{n !} G\left(\langle\Delta E\rangle+h\left(n v_{v}-v\right), \sigma\right)
$$

with the Huang-Rhys factor [79] $S$ and the effective frequency of skeletal intramolecular vibrations $v_{v}$. The inhomogeneous broadening of each vibronic line is characterized by the Gaussian width $\sigma$

$$
G(x, \sigma)=\left[2 \pi \sigma^{2}\right]^{-1 / 2} \exp \left(-\frac{x^{2}}{2 \sigma^{2}}\right) .
$$

Global fits of both the absorption and emission lines were done for the normalized bands based on the measured absorption extinction coefficient $\epsilon(\bar{v})$ [or $\bar{v} \epsilon(\bar{v})$ ] and the emission band shape $I_{\mathrm{em}}(\bar{v}) / \bar{v}$ accounting for the energy gap dependence of the transition dipole $[22,71,80]\left[I_{\mathrm{em}}(\bar{v})\right.$ is the emission flux]. The vibronic progression was modeled with the same value of the Huang-Rhys factor [3,79] $S=2.51$ and fixed effective frequency [44] $\bar{v}_{v}=1300 \mathrm{~cm}^{-1}$ of intramolecular vibrations of DCVA for all absorption and emission bands.

Two major results come from the band shape analysis based on Eqs. (18) and (19). First, we find a significant reorganization energy of electron transfer in nonpolar cyclohexane, with the magnitude on par with reorganization energies reported in weakly polar solvents [11]. Second, we report

$$
\sigma_{1}<\sigma_{2}
$$

in violation of rule III. Here, $\sigma_{1}$ refers to the inhomogeneous, solvent-induced broadening of the charge-transfer absorption line and $\sigma_{2}$ is the corresponding broadening of the emission line. According to the formal definition of the reorganization energy in terms of the Gaussian variance [Eq. (2)], we in fact find

$$
\lambda_{1}<\lambda_{2}
$$

This result is interpreted as arising from contraction of the solvation shell of the donor-acceptor complex in the chargeseparated state.

The difference of the first absorption and emission spectral moments provides us with the solvent-induced Stokes shift $h \Delta v_{s}^{\text {St }}$ [Eq. (15)] and the corresponding Stokes-shift reorganization energy $\lambda^{\mathrm{St}}=h \Delta v_{s}^{\mathrm{St}} / 2$. All three reorganization energies are not equal, in violation of rules II and III. Similar inequality between three alternative definitions of the reorganization energy was found in non-Gaussian models of electron transfer $[27,49]$, which can be realized for reactions with altering polarizability of the donor-acceptor complex, Duschinsky rotations [55], etc. The important consequence of theses non-Gaussian models is the replacement of the Gaussian broadening functions in Eq. (18) with non-Gaussian functions. Therefore the use of Gaussians is an approximation of the current analysis. The present experimental results connect to the previous solvation dynamics studies $[60,73,74]$ to call for the development of a nonlinear (non-Gaussian) theory of electron transfer in nonpolar solvents. Since such a theoretical framework currently does not exist, our estimates 


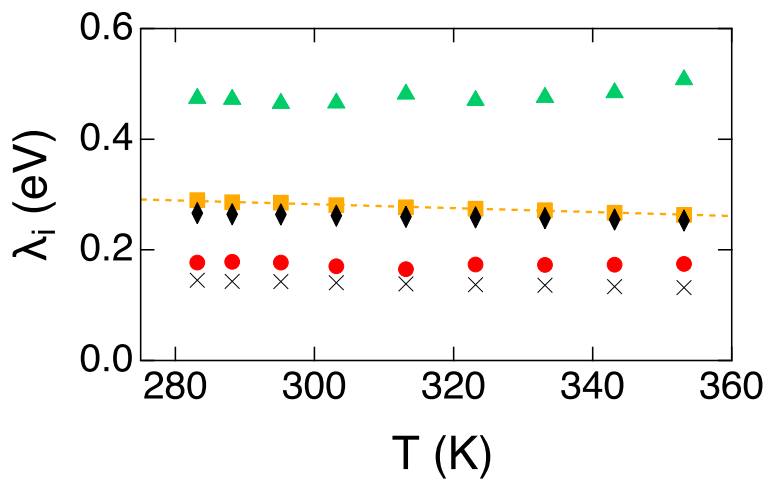

FIG. 4. Reorganization energies for absorption (red) and emission (green) transitions and the solvent-induced Stokes shift $h \Delta \nu_{s}^{\mathrm{St}}$ (orange) vs $T$. The dashed line drawn through the Stokes shift data is the difference of fitting functions in Fig. 3. Black crosses indicate the solvent part of the Stokes-shift reorganization energy $\lambda^{\mathrm{St}}=h \Delta v_{s}^{\mathrm{St}} / 2$, while black diamonds refer to the total (solvent and classical intramolecular) Stokes-shift reorganization energy $\lambda_{\mathrm{cl}}^{\mathrm{St}}[\mathrm{Eq}$. (23)].

of reorganization energies based on the Gaussian theory of fluctuating induction interactions [19] are of limited reach.

Figure 3 shows temperature dependent transition energies $h v^{\text {abs/em }}$ calculated from spectral fits. These energies are connected to first spectral moments of charge-transfer bands by the relation

$$
\langle v\rangle^{\mathrm{abs} / \mathrm{em}}=v^{\mathrm{abs} / \mathrm{em}} \pm v_{v} S,
$$

where + and - refer to absorption and emission, respectively, and $v^{\text {abs/em }}$ define the solvent-induced Stokes shift in Eq. (15). The experimental values $v^{\text {abs/em }}$ are fitted to Eqs. (11) and (13) with $\Delta m=14 \mathrm{D}$ adopted in Eq. (12) according to the distance between centers of charge localization in the charge-separated state (Fig. 1) [71]. The solvent-induced shift of the emission line is produced by Eq. (13). The fit of the experimental first spectral moments to Eqs. (11) and (13) requires two different solute effective radii, $a_{1}=5.08 \AA$ and $a_{2}=3.92 \AA$. These results point to a substantial contraction of the solvation shell upon charge transfer $[60,74]$. The reorganization energies calculated from Eq. (14) with the PY structure factor are equal to $\lambda_{1}=0.12 \mathrm{eV}$ and $\lambda_{2}=1.1 \mathrm{eV}$ at $T=303 \mathrm{~K}$, which should be compared to, correspondingly, $0.17 \mathrm{eV}$ and $0.47 \mathrm{eV}$ from the band shape analysis (Fig. 4). The numbers for emission suggest that the extent of the solute size contraction might be overestimated. We remind here that Eq. (14) is derived within a linear (Gaussian) model of solvation [19] and does not incorporate the non-Gaussian variation of $\lambda$ in a consistent fashion.

As a matter of comparison, the analysis of charge-transfer solvatochromism in polar solvents within the Onsager model of dipole solvation $[62,81,82]$ leads to the average solute radius of $a \simeq 5.37 \AA$ (Fig. 11). An effective radius for dipolar solute-solvent interactions, scaling as $\propto r^{-3}$, should be defined by taking $n=2 \times 3=6$ in Eq. (17). According to this recipe, it is expected to be larger than the effective radius for more short-ranged induction interactions in nonpolar solvents [19], as indeed found from our analysis. We also find that fits of $v^{\text {abs/em }}$ in Fig. 3 produce a nonzero offset of the gas-phase energies $h\left(v_{0}^{\mathrm{abs}}-v_{0}^{\mathrm{em}}\right) \simeq 0.24 \mathrm{eV}$, which is attributed to the Stokes shift due to classical intramolecular vibrational reorganization. This offset is subtracted from the difference of average transition energies [Eq. (15)] to report the solvent-induced Stokes shift shown by orange points in Fig. 4.

The existence of a nonzero $h\left(v_{0}^{\mathrm{abs}}-v_{0}^{\mathrm{em}}\right)$ indicates a possibility of a classical vibrational reorganization energy contributing to $\lambda_{i}$ and to the corresponding Stokes shift. Measurements of spectra at varying temperature allow us to separate this effect by subtracting the gas-phase offset and producing the solvent-induced Stokes shift $h \Delta v_{s}^{\text {St }}$ [Eq. (15)] and the solvent reorganization energy (black crosses in Fig. 4). This value, $\simeq 0.14 \mathrm{eV}$, is the most reliable estimate of the solvent reorganization energy produced by our measurements. At the same time, the reorganization energies $\lambda_{i}$ contain an unspecified small contribution from classical internal vibrations. The total Stokes shift due to solvent and these intramolecular vibrations can be used to characterize the corresponding reorganization energy due to classical solvent and internal modes

$$
\lambda_{\mathrm{cl}}^{\mathrm{St}}=h\left(v^{\mathrm{abs}}-v^{\mathrm{em}}\right) / 2 .
$$

Those values are shown by black diamonds in Fig. 4. The three alternative definitions of the reorganization energy satisfy the inequality

$$
\lambda_{1}<\lambda_{\mathrm{cl}}^{\mathrm{St}}<\lambda_{2} .
$$

Exactly this inequality follows from non-Gaussian models of thermal electron transfer [27] and corresponding static [43] and dynamic [46] band shapes. The appearance of this inequality from our analysis suggests a universal character of these non-Gaussian models offering a possibility of mapping them on the problem of non-Gaussian statistics of electron transfer in nonpolar solvents.

As mentioned above, two separate values for the solute size $a_{i}$ lead to substantially different reorganization energies $\lambda_{i}$, which separately follow from the band shape fitting and are shown in Fig. 4. The individual points $\lambda_{i}(T)$ calculated from the fits at different temperatures are more scattered than the average energies shown in Fig. 3 and cannot be used to establish the temperature dependence of the reorganization energies (Table II). Given a strong dependence of the effective solute radius on the local density profile, we anticipate that testing the explicit temperature dependence $\lambda_{i} \propto \beta$, which is strictly valid at constant density, will be challenging based on experiments done at constant pressure. Thermal expansion of the solvent propagates into a corresponding thermal alteration of the effective solute radius. Nevertheless, the temperature dependence of the Stokes shift is well reproduced by the theory given that individual absorption and emission shifts shown in Fig. 3 are fitted with temperature-independent effective solute radii (the sensitivity of $\left\langle\Delta F_{e}\right\rangle \propto a^{-3}$ to the details of the density profile is much lower compared to $\lambda \propto a^{-9}$ ). The energy scale used in Fig. 4 does not allow one to fully appreciate the temperature drop of $h v_{s}^{\text {St }}$, but it follows the anticipated $T^{-1}$ scaling with temperature (Fig. 10).

To the best of our knowledge, this is the first direct measurement of the solvent reorganization energy of electron transfer in a nonpolar solvent and its temperature dependence based on spectroscopic measurements of both absorption and 
emission charge-transfer bands. A large value of the reorganization energy, $\simeq 0.87 \mathrm{eV}$, was reported from the top of the inverted Marcus parabola for bimolecular reactions between an excited fluorophore and a number of quenchers in cyclohexane by Chen et al. [37]. The reported reorganization energy might still include an unknown intramolecular vibrational component. More detailed fitting of the energy gap laws, including quantum intramolecular modes, was recently reported by Holroyd and Miller [13]. They studied the reaction of electron attachment to naphthalene and t-stilbene in tetramethylsilane and 2,2,4-trimethylpentane and reported solvent reorganization energies of $0.31 \mathrm{eV}$ (naphthalene) and $0.14 \mathrm{eV}$ (t-stilbene). These latter results are consistent with the findings presented here. Much smaller reorganization energies ( $\simeq 0.02 \mathrm{eV}$ in $\mathrm{CCl}_{4}$ ) reported by Fleming and co-workers [60] were measured with a quadrupolar chromophore, in contrast to a highly dipolar charge-transfer complex used here.

We need to stress that the theoretical framework outlined in Eqs. (7)-(12) and given in more detail in Appendix A is a linear theory leading to the Gaussian probability for the rate constant in Eq. (1) and, with account for Eq. (5), to an inverted parabola for the energy gap law. As mentioned above, this framework also requires using Gaussian broadening functions in the band shape analysis [Eq. (18)]. The density contraction resulting in different reorganization energies in two charge-transfer states is fundamentally a nonlinear phenomenon producing a non-Gaussian statistics of the energy gap and a skewed inverted parabola for the energygap law [27]. It can be thought of as an analog of altering force constants in intramolecular reorganization [55] since a highly nonlinear alteration of an effective repulsion potential is projected, in liquid-state theories, to an effective (statedependent) Gaussian susceptibility [83]. An extension of the theory beyond the linear framework is still required for a quantitative analysis.

\section{CONCLUSIONS}

The band shape analysis of charge-transfer absorption and emission lines in nonpolar cyclohexane demonstrates a principal departure from standard theories of electron transfer developed for polar solvents [10]. These classical theories predict that the solvent reorganization energy is proportional to the Pekar factor [84] $c_{0}=\epsilon_{\infty}^{-1}-\epsilon_{s}^{-1}$. According to this prediction, the reorganization energy should drop to nearly zero values when the static dielectric constant $\epsilon_{s}$ becomes close to $\epsilon_{\infty} \simeq n_{D}^{2}$ in nonpolar solvents. In contrast to this prediction, we find quite substantial reorganization energies, $\lambda_{1} \simeq 0.17 \mathrm{eV}$ and $\lambda_{2} \simeq 0.47 \mathrm{eV}$, in nonpolar cyclohexane. The reorganization energies from the band shape analysis are roughly consistent with the analytical theory based on the idea of fluctuating induction interaction of the charge-transfer complex with the nonpolar solvent. A larger reorganization energy of the emission band requires a smaller effective solute radius affected by contraction of the solvation shell upon charge transfer.

Taken together, the results of the band shape analysis violate all three fluctuation-dissipation relations [rules I-III in Eqs. (2)-(4)] on which the Gaussian models of hopping conductivity and the theory of molecular electron transfer are based. Violations of the FDT have been anticipated for systems far from equilibrium [85] when the rules of sampling established by the Gibbs ensemble do not apply anymore. The situation is different here. The violation of the FDT comes from the microscopic nature of the solute-solvent interaction energy, which, due to its short range, does not follow the statistical rules derived for macroscopic and quasimacroscopic thermally fluctuating collective coordinates.

\section{ACKNOWLEDGMENTS}

This research was supported by the US Department of Energy, Office of Science, Office of Basic Energy Sciences, under Award DE-SC0015641.

\section{APPENDIX A: DERIVATION OF ANALYTICAL RESULTS}

Liquid-state perturbation theory $[19,69]$ is used to evaluate the average and variance of the instantaneous solvent-induced shift of the transition energy

$$
\Delta F_{e}=F_{e 2}-F_{e 1} .
$$

The perturbation theory evaluates averages $\left\langle\Delta F_{e}\right\rangle_{i}$ in terms of the reference average $\langle\ldots\rangle$ over the equilibrium configurations consistent with the state in which the induction solute-solvent interaction is turned off. In this representation, all the dependence on the electronic state of the chargetransfer complex is shifted to the dipole moments

$$
\left\langle\Delta F_{e}\right\rangle_{i}=-\delta m^{2}\langle U\rangle-\left(2 m_{i}^{2} / \delta m^{2}\right) \lambda,
$$

where $\delta m^{2}=m_{2}^{2}-m_{1}^{2}, U$ is from Eqs. (7) and (8), and the reorganization energy is given by Eq. (10) to which we substitute $\delta m^{2}=(\Delta m)^{2}$ based on the assumption $\mathbf{m}_{1} \approx 0$. Adopting $\mathbf{m}_{1}=0$, the shift of the absorption line becomes

$$
\left\langle\Delta F_{e}\right\rangle_{1}=-(\Delta m)^{2}\langle U\rangle .
$$

Correspondingly, we obtain for the emission line

$$
\left\langle\Delta F_{e}\right\rangle_{2}=-(\Delta m)^{2}\langle U\rangle-2 \lambda
$$

where in both equations

$$
\langle U\rangle=\frac{4 \pi}{3 a^{3}} \alpha \rho, \quad a^{-3}=3 \int_{0}^{\infty} \frac{d r}{r^{4}} g_{0 s}(r) .
$$

Here, the effective radius of the solute $a$ corresponds to $n=6$ in Eq. (17). The use of the Clausius-Mossotti equation converts Eq. (A5) to

$$
\langle U\rangle=\frac{\epsilon_{\infty}-1}{\epsilon_{\infty}+2} \frac{1}{a^{3}} .
$$

The local density profile determined by $g_{0 s}(r)$ is different in each charge-transfer state [48] and one obtains two different values of the effective radius $a_{i}$ to produce $\Delta F_{e}\left(a_{i}\right)$ in Eq. (12).

The reorganization energy requires the variance of $U$ in Eq. (10). The variance can be written [19] as the reciprocalspace integral involving the density-density structure factor [29] $S(k)$

$$
\left\langle(\delta U)^{2}\right\rangle=\rho \int \frac{d \mathbf{k}}{(2 \pi)^{3}} \tilde{v}(\mathbf{k})^{2} S(k),
$$


TABLE I. Properties of cyclohexane used in the calculations.

\begin{tabular}{cccccc}
\hline \hline Property & $\sigma_{s}, \AA$ & $\eta$ & $\alpha, \AA^{3}$ & $\epsilon_{\infty}$ & $\alpha_{p} \times 10^{3}, \mathrm{~K}^{-1}$ \\
\hline & 5.635 & 0.519 & 11.0 & 2.03 & 1.23 \\
\hline \hline
\end{tabular}

where $\tilde{v}(\mathbf{k})$ is the spatial Fourier transform of the interaction energy

$$
\tilde{v}(\mathbf{k})=4 \pi \alpha k^{3}\left[f_{0}(k a)-f_{2}(k a) P_{2}(\hat{\mathbf{k}} \cdot \hat{\mathbf{m}})\right]
$$

and

$$
f_{n}(k a)=\int_{k a}^{\infty} \frac{d x}{x^{4}} j_{n}(x),
$$

$j_{n}(x)$ is the $n$ th-order spherical Bessel function.

Equation (A7) is further simplified by integration over the angles of $\mathbf{k}$ vectors

$$
\left\langle(\delta U)^{2}\right\rangle=8 \alpha^{2} \rho \int_{0}^{\infty} d k k^{8}\left[f_{0}^{2}(k a)+\frac{1}{5} f_{2}^{2}(k a)\right] S(k) .
$$

The reorganization energy is obtained by substituting Eq. (A10) to Eq. (10)

$$
\lambda=\frac{4 \beta \alpha^{2}(\Delta m)^{4}}{a^{9}} \rho J,
$$

where

$$
J=a^{9} \int_{0}^{\infty} d k k^{8}\left[f_{0}^{2}(k a)+\frac{1}{5} f_{2}^{2}(k a)\right] S(k) .
$$

Given that $S(k)$ is a function of $k \sigma_{s}\left(\sigma_{s}\right.$ is the solvent diameter), the integral $J$ is a function of $a / \sigma_{s}$ and the solvent density affecting the density-density structure factor $S(k)$.

Calculations were done for cyclohexane as a solvent, with parameters required for calculations listed in Table I [86]. The hard-sphere diameter $\sigma_{s}$ was calculated from the isothermal compressibility $\beta_{T}$ of cyclohexane by using the generalized van der Waals equation of state [86]. The value $\sigma_{s}=5.635 \AA$ listed in Table I is consistent with hard-sphere diameters of other nonpolar liquids, and it falls on a master curve [87] for $\sigma_{s}$ vs the $b^{1 / 3}: b^{1 / 3}=0.9337+0.5828 \sigma_{s}+0.03315 \sigma_{s}^{2}$, where $b$ is the second van der Waals constant in $\mathrm{cm}^{3} / \mathrm{mol}$ and $\sigma_{s}$ is in $\AA$. The packing fraction $\eta=(\pi / 6) \rho \sigma_{s}^{3}$ was used to define the PY structure factor from the solution for the fluid of hard spheres [69]. Since the PY solution does not reproduce the experimental compressibility of the liquid, the packing fraction was adjusted in the PY solution to ensure the condition $S(0)=\rho k_{\mathrm{B}} T \beta_{T}$. This requires a downward scaling of $\eta$ to $\eta=0.4613$. However, the packing fraction listed in Table I was used in the rest of calculations. Its dependence on temperature due to solvent expansion was produced by using the isobaric expansivity $\alpha_{p}$ (Table I)

$$
\eta(T)=\eta\left(T_{0}\right)\left[1-\alpha_{p}\left(T-T_{0}\right)\right] .
$$

In addition, the temperature dependence of $\epsilon_{\infty}(T)$ is from Ref. [88].

Figure 5 illustrates the scaling $\lambda \propto a^{-9}$ introduced in Eq. (A11) by plotting $a^{9} \lambda$ versus $a$ at different $T$. There are some low-amplitude oscillations coming from the integral $J$ in Eq. (A12), but the scaling is mostly maintained. Figure 6

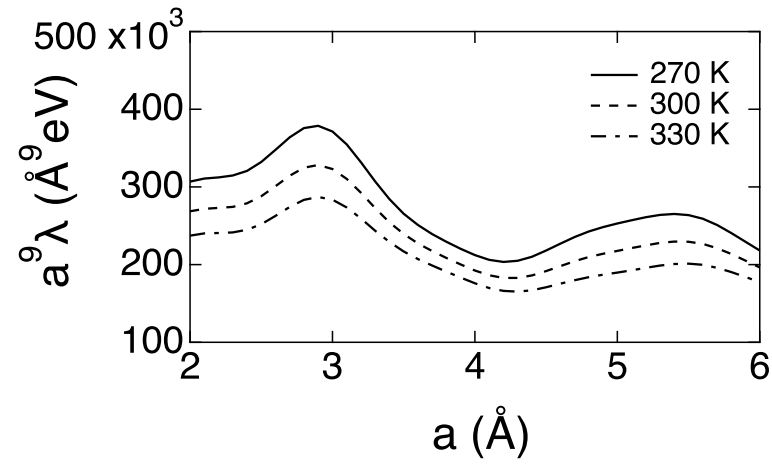

FIG. 5. $a^{9} \lambda$ calculated from Eq. (A11) at the temperature listed in the plot.

illustrates proportionality of the reorganization energy to the inverse temperature, $\lambda \propto T^{-1}$. The product $T \lambda(T)$ in Fig. 6 changes little when temperature is varied.

The average induction energy in Eqs. (A3) and (A5) scales linearly with $\alpha(\Delta m)^{2}$, while the reorganization energy scales quadratically with this factor. One can rearrange Eq. (A11) for the reorganization energy is terms of Eq. (14) with

$$
f\left(\eta, a / \sigma_{s}\right)=\frac{3}{8 \pi \eta}\left(\frac{\sigma_{s}}{a}\right)^{3} J .
$$

To provide a numerical estimate, the function $f(\eta, a)$ was calculated by performing the numerical integration in Eqs. (A12) and (A14) with the PY $S(k)$. The function was fitted to a polynomial

$$
f(\eta, a)=\sum_{i=0}^{2} \frac{C_{i}(\eta)}{a^{3+i}}
$$

in which the functions $C_{i}(\eta)$ are given in the form

$$
C_{i}(\eta)=A_{i} \exp \left[-B_{i} \eta\right]
$$

with $A_{i}=0.5047,0.2463,-0.1545$ and $B_{i}=12.14,3.33$, 5.11 for $i=0,1,2$.

\section{APPENDIX B: BAND SHAPE ANALYSIS}

Global fit of both absorption and emission bands to the same set of parameters was done at each temperature. The extinction coefficient $\epsilon(\bar{v}) \propto \mathrm{FC}(\bar{v})$ [Eqs. (18) and (19)] was

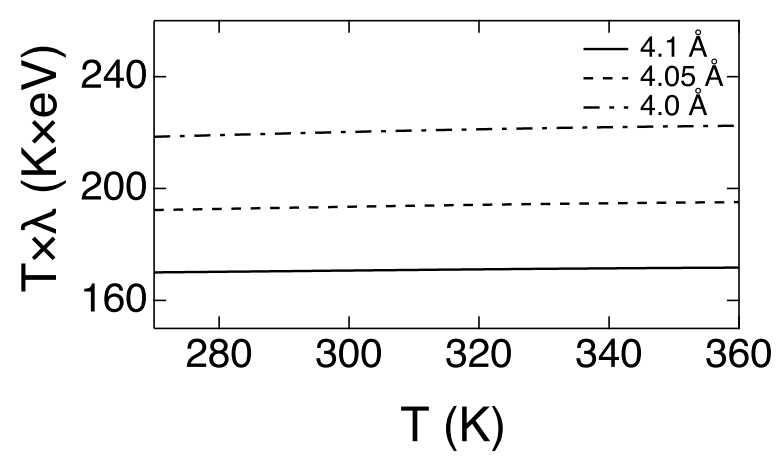

FIG. 6. $T \times \lambda(T)$ calculated from Eq. (A11) at the values of the solute effective radius $a$ listed in the plot. 


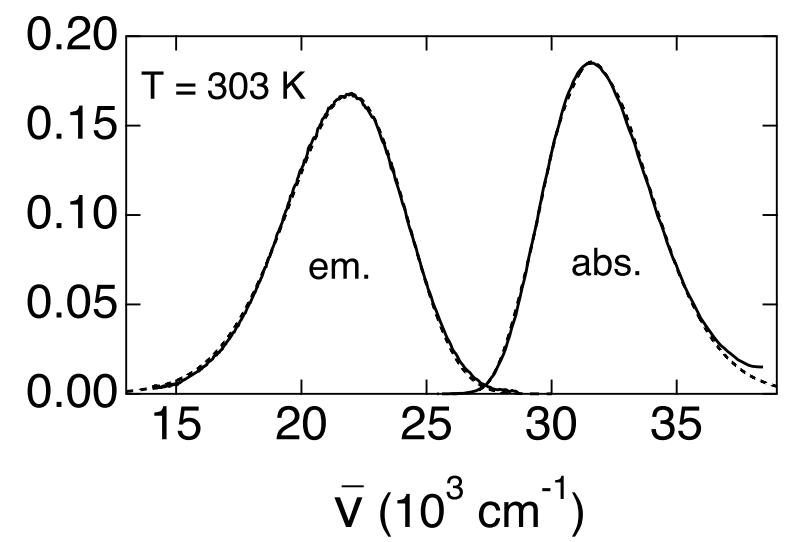

FIG. 7. Fits (dashed lines) of absorption (abs.) and emission (em.) band shapes at $T=303 \mathrm{~K}$ (solid lines).

fitted to the vibronic progression of equally spaced lines characterized by the vibrational frequency [44] $\bar{v}_{v}=1300 \mathrm{~cm}^{-1}$ and the Huang-Rhys factor $S=2.51$. A similar functional form, with separate values of $\langle\Delta E\rangle_{2}$ and $\sigma_{2}$ for the emission transition, is used for the reduced emission band shape $[22,78,80] I_{\mathrm{em}}(\bar{v}) / \bar{v}$, where $I_{\mathrm{em}}(\bar{v})$ is the photon flux. The Huang-Rhys factor $S$ is fixed at $S=2.51$ in all fits after preliminary runs using it as an independent fitting parameter. Examples of fits are shown in Fig. 7 and the fit results are listed in Table II. The inhomogeneous width is converted to the solvent reorganization energy through the relation $\sigma_{i}^{2}=$ $2 \lambda_{i} k_{\mathrm{B}} T$.

The appearance of $I_{\mathrm{em}}(\bar{v}) / \bar{v}$ for the emission band shape accounts for the dependence of the transition dipole on transition energy $[22,52,80] M_{12} \propto \bar{v}^{-1}$. In the same approximation, the extinction coefficient $\epsilon(\bar{v})$ needs to be replaced with $\bar{v} \epsilon(\bar{v})$ to extract the Franck-Condon density [52]. This approach is sometimes less robust from the practical standpoint since it enhances the high-frequency noise in the spectra often affected by locally excited states [45]. Nevertheless, this protocol was also adopted in our analysis (Fig. 8), and the results of band shape fitting are listed in the third column of Table II. In Fig. 9, we compare the first spectral moments $h v^{\text {abs }}$ versus $T$ from two fitting procedures and list Stokes shifts in the 6th column in Table II. Figure 10 shows the solvent-induced

TABLE II. Reorganization energies from absorption and emission widths $\lambda_{i}\left(\mathrm{~cm}^{-1}\right)$ and half of the Stokes shift $h \Delta v^{\mathrm{St}} / 2\left(\mathrm{~cm}^{-1}\right)$ from the band shape analysis.

\begin{tabular}{lccccc}
\hline \hline$T, \mathrm{~K}$ & $\lambda_{1}$ & $\lambda_{1}{ }^{\mathrm{a}}$ & $\lambda_{2}$ & $h \Delta \nu^{\mathrm{St}} / 2$ & $h \Delta \nu^{\mathrm{St}} / 2^{\mathrm{a}}$ \\
\hline 283.15 & 1427.9 & 1670.4 & 3821.6 & 2147.6 & 2214.2 \\
288.15 & 1437.3 & 1635.7 & 3807.7 & 2132.2 & 2198.2 \\
295.15 & 1428.8 & 1625.5 & 3747.2 & 2128.5 & 2194.7 \\
303.15 & 1374.1 & 1610.7 & 3752.9 & 2111.1 & 2178.2 \\
313.15 & 1331.6 & 1597.3 & 3884.1 & 2095.8 & 2163.7 \\
323.15 & 1398.1 & 1594.1 & 3790.4 & 2085.0 & 2152.0 \\
333.15 & 1393.0 & 1586.4 & 3836.6 & 2074.5 & 2141.9 \\
343.15 & 1397.0 & 1572.7 & 3902.3 & 2055.9 & 2123.3 \\
353.15 & 1407.0 & 1578.4 & 4091.8 & 2040.7 & 2108.4 \\
\hline \hline
\end{tabular}

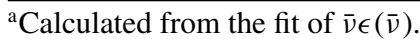

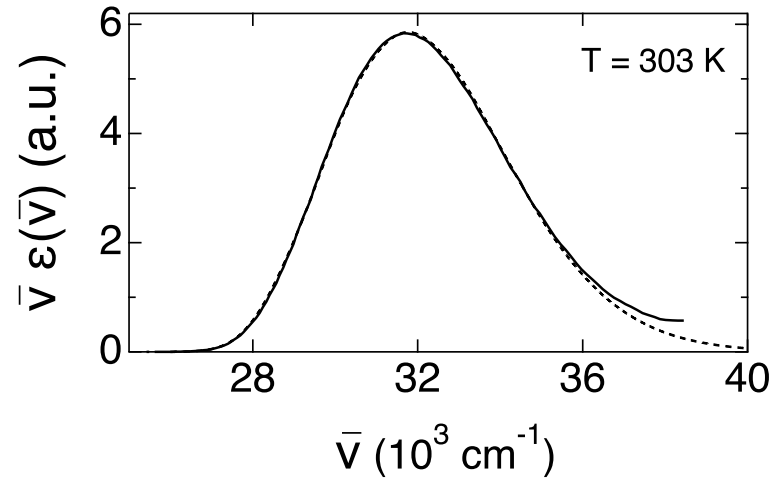

FIG. 8. Fit (dashed line) of the absorption (solid line) band shape at $T=303 \mathrm{~K}$. The experimental extinction coefficient is multiplied by $\bar{v}$ and the function $\bar{v} \epsilon(\bar{v})$ is fitted to the right-hand side of Eq. (18).

Stokes shift calculated by two procedures. Overall, the adoption of $\bar{v} \epsilon(\bar{v})$ in the fitting protocol amounts to an upward shift in both $\lambda_{1}$ and the overall Stokes shift. However, this change does not affect any of our conclusions.

The average absorption and emission energies $h\langle v\rangle^{\mathrm{abs} / \mathrm{em}}=$ $\langle\Delta E\rangle_{i}$ (first spectral moments) are used to determine the transition energies $v^{\text {abs/em }}$ according to Eq. (22), with $h v^{\text {abs/em }}(T)$ shown in Fig. 3. They are fitted to Eqs. (11) and (13) in which the solute radius $a_{i}$ is considered as a fitting parameter. Since the PY solution might be too simplified for the experimental conditions, the function $f\left(\eta, a / \sigma_{s}\right)$ in Eq. (A14) was taken as a temperature-independent fitting parameter.

This fit produces $a_{i}$ and the vacuum energies $v_{0}^{\mathrm{abs} / \mathrm{em}}$. They are shifted by $0.24 \mathrm{eV}$ suggesting that classical intramolecular modes contribute to the vacuum Stokes shift. When the same analysis is performed on the data obtained from fitting $\bar{v} \epsilon(\bar{v})$ for the absorption lines, the vacuum Stokes shift becomes $0.26 \mathrm{eV}$. This component is subtracted from $\langle v\rangle^{\mathrm{abs}}-\langle v\rangle^{\mathrm{em}}$ in both cases to obtain the solvent-induced Stokes shift plotted in Fig. 10. A somewhat larger vacuum intercept makes the solvent-induced Stokes shift lower in the second protocol adopted for fitting the absorption lines. Note that the values of the Stokes shift listed in Table II are raw results of the band shape analysis, which include both the solvent-induced and classical vibrational contributions to the Stokes shift.

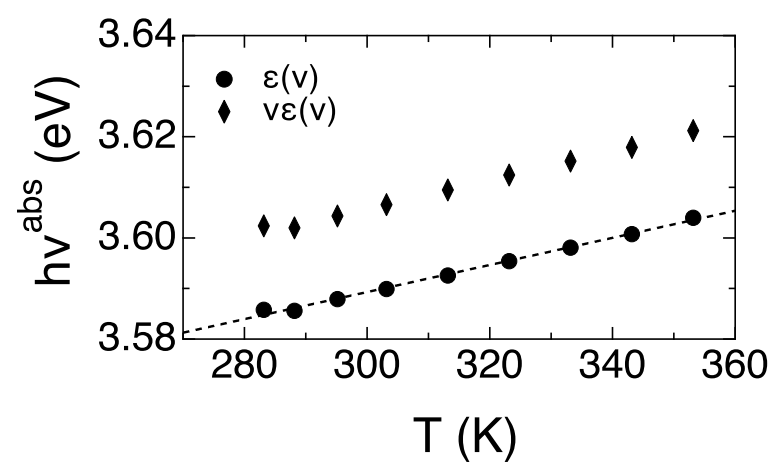

FIG. 9. $h v^{\text {abs }}(T)$ from fitting absorption bands (points). Two protocols are used in the fits: fitting the extinction coefficient to Eq. (18) and fitting $\bar{v} \epsilon(\bar{v})$ to the right-hand side of Eq. (18). The dashed line is a linear fit through the points. 


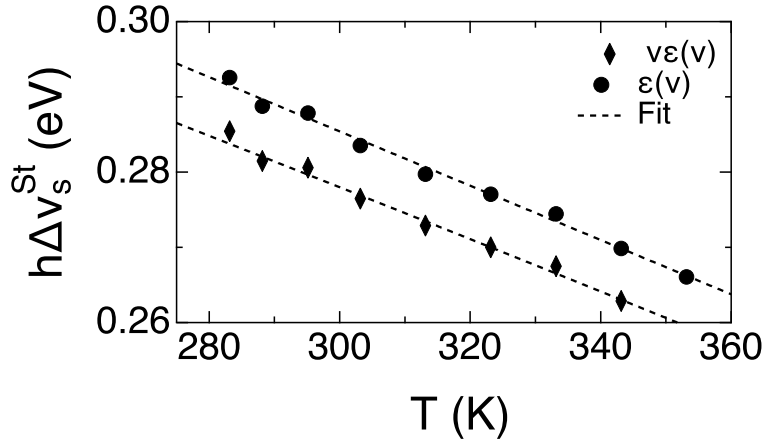

FIG. 10. Solvent-induced Stokes shift from fitting absorption and emission bands (points) vs temperature. The fitting of absorption lines was done by either using the extinction coefficient $\epsilon(\bar{v}) \propto$ $\mathrm{FC}(\bar{v})$ [circles, Eq. (18)] or the product $\bar{v} \epsilon(\bar{v})$ (diamonds). The dashed lines are linear fits through the points.

According to Eq. (A11), the solvent-induced Stokes shift is a decaying function of temperature $\left(h \Delta v_{s}^{\mathrm{St}}=2 \lambda \propto \beta\right.$ in the linear (Gaussian) theory).

An alternative estimate of the effective radius of the DCVA molecule is established from the analysis of the Stokes shift changing with the solvent polarity in terms of the Onsager model of dipole solvation [62]

$$
h \Delta v_{s}^{\mathrm{St}}=\frac{2(\Delta m)^{2}}{a^{3}} F\left(\epsilon_{\infty}, \epsilon_{s}\right),
$$

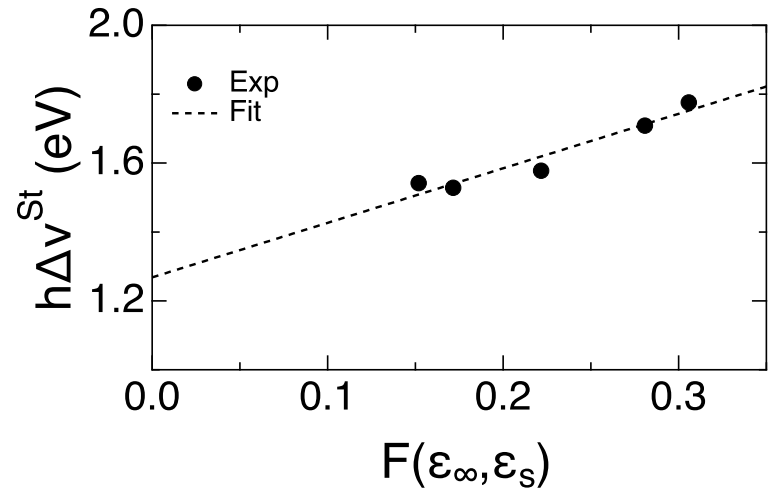

FIG. 11. Dependence of the Stokes shift of DCVA on $F\left(\epsilon_{\infty}, \epsilon_{s}\right)$ [Eq. (B2)] for chloroform, 2-methyltetrahydrofurane, 1,2-dichloroethane, butyronitrile, acetonitrile [71] (in the order of increasing $F\left(\epsilon_{\infty}, \epsilon_{s}\right)$ ). The dashed line is a linear fit through the experimental points.

where

$$
F\left(\epsilon_{\infty}, \epsilon_{s}\right)=\frac{\epsilon_{s}-1}{2 \epsilon_{s}+1}-\frac{\epsilon_{\infty}-1}{2 \epsilon_{\infty}+1} .
$$

The plot of $h \Delta v^{\mathrm{St}}=h\langle v\rangle^{\mathrm{abs}}-h\langle v\rangle^{\mathrm{em}}$ vs $F\left(\epsilon_{\infty}, \epsilon_{s}\right)$ for a number of polar solvents [71] is shown in Fig. 11. The effective radius for $\Delta m=14 \mathrm{D}$ is $a=5.37 \AA$.
[1] P. Anderson, Absence of diffusion in certain random lattices, Phys. Rev. 109, 1492 (1958).

[2] H. Böttger and V. V. Bryksin, Hopping Conduction in Solids (VCH, Weinheim, 1985).

[3] V. May and O. Kühn, Charge and Energy Transfer Dynamics in Molecular Systems, 3rd ed. (Wiley-VCH, Berlin, 2011).

[4] D. G. Nicholls and S. J. Ferguson, Bioenergetics 3 (Academic Press, London, 2002).

[5] A. J. Hoff and J. Deisenhofer, Photophysics of photosynthesis, Phys. Rep. 287, 1 (1997).

[6] H. B. Gray and J. R. Winkler, Long-range electron transfer, Proc. Natl. Acad. Sci. 102, 3534 (2005).

[7] G. Yu, J. Gao, J. C. Hummelen, F. Wudl, and A. J. Heeger, Polymer photovoltaic cells: Enhanced efficiencies via a network of internal donor-acceptor heterojunctions, Science 270, 1789 (1995).

[8] J. Benduhn, K. Tvingstedt, F. Piersimoni, S. Ullbrich, Y. Fan, M. Tropiano, K. A. McGarry, O. Zeika, M. K. Riede, C. J. Douglas, S. Barlow, S. R. Marder, D. Neher, D. Spoltore, and $\mathrm{K}$. Vandewal, Intrinsic non-radiative voltage losses in fullerenebased organic solar cells, Nat. Energy 2, 297 (2017).

[9] M. Azzouzi, J. Yan, T. Kirchartz, K. Liu, J. Wang, H. $\mathrm{Wu}$, and $\mathrm{J}$. Nelson, Nonradiative Energy Losses in BulkHeterojunction Organic Photovoltaics, Phys. Rev. X 8, 031055 (2018).

[10] R. A. Marcus and N. Sutin, Electron transfer in chemistry and biology, Biochim. Biophys. Acta 811, 265 (1985).

[11] P. Chen and T. J. Meyer, Medium effects on charge transfer in metal complexes, Chem. Rev. 98, 1439 (1998).
[12] V. Garg, G. Kodis, M. Chachisvilis, M. Hambourger, A. L. Moore, T. A. Moore, and D. Gust, Conformationally constrained macrocyclic diporphyrin-fullerene artificial photosynthetic reaction center, J. Am. Chem. Soc. 133, 2944 (2011).

[13] R. A. Holroyd and J. R. Miller, Rate vs free energy change for attaching highly-mobile electrons to molecules in nonpolar liquids, J. Phys. Chem. B 123, 9206 (2019).

[14] C. Nançoz, C. Rumble, A. Rosspeintner, and E. Vauthey, Bimolecular photoinduced electron transfer in non-polar solvents beyond the diffusion limit, J. Chem. Phys. 152, 244501 (2020).

[15] G. Angulo and A. Rosspeintner, Bimolecular photo-induced electron transfer enlightened by diffusion, J. Chem. Phys. 153, 040902 (2020).

[16] V. Coropceanu, J. Cornil, D. A. da Silva Filho, Y. Olivier, R. Silbey, and J.-L. Brédas, Charge transport in organic semiconductors, Chem. Rev. 107, 926 (2007).

[17] T. Kim, W. Kim, H. Mori, A. Osuka, and D. Kim, Solvent and structural fluctuations induced symmetry-breaking charge transfer in a porphyrin triad, J. Phys. Chem. C 122, 19409 (2018).

[18] J. Zhao, Y. Li, G. Yang, K. Jiang, H. Lin, H. Ade, W. Ma, and H. Yan, Efficient organic solar cells processed from hydrocarbon solvents, Nat. Energy 1, 15027 (2016).

[19] D. V. Matyushov, Electron transfer in nonpolar media, Phys. Chem. Chem. Phys. 22, 10653 (2020).

[20] R. A. Marcus, Relation between charge transfer absorption and fluorescence spectra and the inverted region, J. Phys. Chem. 93 3078 (1989). 
[21] N. S. Hush and J. R. Reimers, Solvent effects on metal to ligand charge transfer excitations, Coord. Chem. Rev. 177, 37 (1998).

[22] I. R. Gould, D. Noukakis, L. Gomes-Jahn, R. H. Young, J. L. Goodman, and S. Farid, Radiative and nonradiative electron transfer in contact radical-ion pairs, Chem. Phys. 176, 439 (1993).

[23] P. F. Barbara, T. J. Meyer, and M. A. Ratner, Contemporary issues in electron transfer research, J. Phys. Chem. 100, 13148 (1996).

[24] S. Rafiq and G. D. Scoles, From fundamental theories to quantum coherences in electron transfer, J. Am. Chem. Soc. 141, 708 (2019).

[25] R. Kubo, The fluctuation-dissipation theorem, Rep. Prog. Phys. 29, 255 (1966).

[26] R. P. Feynman, R. B. Leighton, and M. Sands, The Feynman Lectures on Physics, Vol. I: Mainly Mechanics, Radiation, and Heat (Addison-Wesley, Reading, MA, 1963).

[27] D. V. Matyushov and G. A. Voth, Modeling the free energy surfaces of electron transfer in condensed phases, J. Chem. Phys. 113, 5413 (2000).

[28] L. Onsager, Reciprocal relations in irreversible processes. II. Phys. Rev. 38, 2265 (1931).

[29] J.-P. Hansen and I. R. McDonald, Theory of Simple Liquids, 4th ed. (Academic Press, Amsterdam, 2013).

[30] J. N. Braddock and T. J. Meyer, Kinetics of the oxidation of $\mathrm{Fe}\left(\mathrm{H}_{2} \mathrm{O}\right)_{6}^{2+}$ by polypyridine complex ruthenium(III). Negative enthalpies of activation, J. Am. Chem. Soc. 95, 3158 (1973).

[31] J. L. Cramer and T. J. Meyer, Unusual activation parameters in the oxidation of hexaaquairon $(2+)$ ion by polypyridine complexes of iron(iii). Evidence for multiple paths for outer-sphere electron transfer, Inorg. Chem. 13, 1250 (1974).

[32] N. Liang, J. R. Miller, and G. L. Closs, Correlating temperature dependence to free-energy dependence of intramolecular longrange electron transfers, J. Am. Chem. Soc. 111, 8740 (1989).

[33] H. B. Kim, N. Kitamura, Y. Kawanishi, and S. Tazuke, Bell-shaped temperature dependence in quenching of excited $\mathrm{Ru}(\mathrm{bpy})_{3}^{2+}$ by an organic acceptor, J. Am. Chem. Soc. 109, 2506 (1987).

[34] M. M. Waskasi, G. Kodis, A. L. Moore, T. A. Moore, D. Gust, and D. V. Matyushov, Marcus bell-shaped electron transfer kinetics observed in an Arrhenius plot, J. Am. Chem. Soc. 138, 9251 (2016).

[35] M. R. Wasielewski, M. P. Niemczyk, W. Svec, and E. B. Pewitt, Dependence of rate constants for photoinduced charge separation and dark charge recombination on the free energy of reaction in restricted-distance porphyrin-quinone molecules, J. Am. Chem. Soc. 107, 1080 (1985).

[36] G. L. Closs and J. R. Miller, Intramolecular long-distance electron transfer in organic molecules, Science 240, 440 (1988).

[37] J. M. Chen, T. I. Ho, and C. Mou, Experimental investigation of excited-state electron-transfer reaction: effects of free energy and solvent on rates, J. Phys. Chem. 94, 2889 (1990).

[38] I. R. Gould, D. Noukakis, L. Gomez-Jahn, J. L. Goodman, and S. Farid, Explanation of the driving-force dependence of return electron transfer in contact radical-ion pairs, J. Am. Chem. Soc. 115, 4405 (1993).

[39] D. M. Guldi, Nanometer scale carbon structures for chargetransfer systems and photovoltaic applications, Phys. Chem. Chem. Phys. 9, 1400 (2007).
[40] M. Kuss-Petermann and O. S. Wenger, Electron transfer rate maxima at large donor-acceptor distances, J. Am. Chem. Soc. 138, 1349 (2016).

[41] A. Das, N. Kamatham, A. Mohan Raj, P. Sen, and V. Ramamurthy, Marcus relationship maintained during ultrafast electron transfer across a supramolecular capsular wall, J. Phys. Chem. A 124, 5297 (2020).

[42] N. Takeda and J. R. Miller, Inverted region in bimolecular electron transfer in solution enabled by delocalization, J. Am. Chem. Soc. 142, 17997 (2020).

[43] D. V. Matyushov and M. D. Newton, Understanding the optical band shape: Coumarin-153 steady-state spectroscopy, J. Phys. Chem. A 105, 8516 (2001).

[44] S. Arzhantsev, K. A. Zachariasse, and M. Maroncelli, Photophysics of trans-4-(Dimethylamino)-4'-cyanostilbene and its use as a solvation probe, J. Phys. Chem. A 110, 3454 (2006).

[45] M. Sajadi, T. Obernhuber, S. A. Kovalenko, M. Mosquera, B. Dick, and N. P. Ernsting, Dynamic polar solvation is reported by fluorescing 4-aminophthalimide faithfully despite H-bonding, J. Phys. Chem. A 113, 44 (2009).

[46] D. V. Matyushov, Time-resolved spectroscopy of polarizable chromophores, J. Chem. Phys. 115, 8933 (2001).

[47] G. Jeanmairet, B. Rotenberg, M. Levesque, D. Borgis, and M. Salanne, A molecular density functional theory approach to electron transfer reactions, Chem. Sci. 10, 2130 (2019).

[48] C. Hartnig and M. T. M. Koper, Molecular dynamics simulations of solvent reorganization in electron-transfer reactions, J. Chem. Phys. 115, 8540 (2001).

[49] D. W. Small, D. V. Matyushov, and G. A. Voth, The theory of electron transfer: What may be missing? J. Am. Chem. Soc. 125, 7470 (2003).

[50] J. Blumberger and M. Sprik, Ab initio molecular dynamics simulation of the aqueous $\mathrm{Ru}^{2+} / \mathrm{Ru}^{3+}$ redox reaction: The Marcus perspective, J. Phys. Chem. B 109, 6793 (2005).

[51] T. Kakitani and N. Mataga, New energy gap laws for the charge separation process in the fluorescence quenching reaction and the charge recombination process of ion pairs produced in polar solvents, J. Phys. Chem. 89, 8 (1985).

[52] D. V. Matyushov and G. A. Voth, Reorganization parameters of electronic transitions in electronically delocalized systems. 2. Optical spectra, J. Phys. Chem. A 104, 6485 (2000).

[53] J. T. Hupp and M. J. Weaver, The driving-force dependence of electrochemical rate parameters: origins of anodic-cathodic asymmetries for metal-aquo redox couples, J. Phys. Chem. 88, 6128 (1984).

[54] G. A. Tsirlina, Y. I. Kharkats, R. R. Nazmutdinov, and O. A. Petrii, Asymmetry of inner-sphere reorganization energy for heterogeneous electron transfer, J. Electroanal. Chem. 450, 63 (1998).

[55] D. V. Matyushov and M. D. Newton, Q-model of electrode reactions: Altering force constants of intramolecular vibrations, Phys. Chem. Chem. Phys. 20, 24176 (2018).

[56] G. Fischer, Vibronic Coupling (Academic Press, London, 1984).

[57] E. A. Carter and J. T. Hynes, Solute-dependent solvent force constants for ion pairs and neutral pairs in a polar solvent, J. Phys. Chem. 93, 2184 (1989).

[58] T. Fonseca and B. M. Ladanyi, Breakdown of linear response for solvation dynamics in methanol, J. Phys. Chem. 95, 2116 (1991). 
[59] D. V. Matyushov and R. Schmid, Optical and radiationless intramolecular electron transitions in nonpolar fluids: Relative effects of induction and dispersion interactions, J. Chem. Phys. 103, 2034 (1995).

[60] D. S. Larsen, K. Ohta, and G. R. Fleming, Three pulse photon echo studies of nondipolar solvation: Comparison with a viscoelastic model, J. Chem. Phys. 111, 8970 (1999).

[61] B.-C. Perng, M. D. Newton, F. O. Raineri, and H. L. Friedman, Energetics of charge transfer reactions in solvents of dipolar and higher order multipolar character. II. Results, J. Chem. Phys. 104, 7177 (1996).

[62] L. Reynolds, J. A. Gardecki, S. J. V. Frankland, and M. Maroncelli, Dipole solvation in nondipolar solvents: Experimental studies of reorganization energies and solvation dynamics, J. Phys. Chem. 100, 10337 (1996).

[63] D. V. Matyushov and G. A. Voth, A perturbation theory for solvation thermodynamics: Dipolar-quadrupolar liquids, J. Chem. Phys. 111, 3630 (1999).

[64] M. B. Zimmt and D. H. Waldeck, Exposing solvent's role in electron transfer reactions: Tunneling pathway and solvation, J. Phys. Chem. A 107, 3580 (2003).

[65] B. M. Ladanyi and M. Maroncelli, Mechanisms of solvation dynamics of polyatomic solutes in polar and nondipolar solvents: A simulation study, J. Chem. Phys. 109, 3204 (1998).

[66] A. A. Milischuk and D. V. Matyushov, Equlibrium solvation in quadrupolar solvents, J. Chem. Phys. 123, 044501 (2005).

[67] E. Lippert, Spectroscopische bestimmung des Dipolmomentes aromatischer Verbindungen im ersten angeregten Singulettzustand, Z. Electrochem. 61, 962 (1957).

[68] E. G. McRae, Theory of solvent effects on molecular electronic spectra. Frequency shifts, J. Phys. Chem. 61, 562 (1957).

[69] C. G. Gray and K. E. Gubbins, Theory of Molecular Liquids, Vol. 1: Fundamentals (Clarendon Press, Oxford, 1984).

[70] P. Pasman, F. Rob, and J. W. Verhoeven, Intramolecular chargetransfer absorption and emission resulting from through-bond interaction in bichromophoric molecules, J. Am. Chem. Soc. 104, 5127 (1982).

[71] T. Mukherjee, N. Ito, and I. R. Gould, Experimental exploration of the Mulliken-Hush relationship for intramolecular electron transfer reactions, J. Phys. Chem. A 115, 1837 (2011).

[72] M. Dinpajooh and D. V. Matyushov, Free energy of ion hydration: Interface susceptibility and scaling with the ion size, J. Chem. Phys. 143, 044511 (2015).
[73] J. T. Fourkas, A. Benigno, and M. Berg, Completely non-polar solvation as a probe of mechanical relaxation in glass-forming liquids, J. Non-Cryst. Sol. 172-174, 234 (1994).

[74] M. Berg, Viscoelastic continuum model of nonpolar solvation: 1. Implementations for multiple time scales in liquid dynamics, J. Phys. Chem. A 102, 17 (1998).

[75] B. Bagchi, Molecular theory of nonpolar solvation dynamics, J. Chem. Phys. 100, 6658 (1994).

[76] M. Bixon and J. Jortner, Electron transfer - From isolated molecules to biomolecules, Adv. Chem. Phys. 106, 35 (1999).

[77] T. Kumpulainen, B. Lang, A. Rosspeintner, and E. Vauthey, Ultrafast elementary photochemical processes of organic molecules in liquid solution, Chem. Rev. 117, 10826 (2017).

[78] J. S. Beckwith, C. A. Rumble, and E. Vauthey, Data analysis in transient electronic spectroscopy - an experimentalist's view, Int. Rev. Phys. Chem. 39, 135 (2020).

[79] K. Huang and A. Rhys, Theory of light absorption and nonradiative transitions in F-centres, Proc. R. Soc. London Ser. A 204, 406 (1950).

[80] D. V. Matyushov and M. D. Newton, Optical absorption by charge-transfer molecules, J. Phys. Chem. B 123, 6564 (2019).

[81] L. Onsager, Electric moments of molecules in liquids, J. Am. Chem. Soc. 58, 1486 (1936).

[82] D. V. Matyushov, Dipole solvation in dielectrics, J. Chem. Phys. 120, 1375 (2004).

[83] D. Chandler, Gaussian field model of fluids with an application to polymeric fluids, Phys. Rev. E 48, 2898 (1993).

[84] S. I. Pekar, Research in Electron Theory of Crystals (USAEC, Washington, D.C., 1963).

[85] J. Kurchan, In and out of equilibrium, Nature (London) 433, 222 (2005).

[86] R. Schmid and D. V. Matyushov, Entropy of attractive forces and molecular nonsphericity in real liquids: A measure of structural ordering, J. Phys. Chem. 99, 2393 (1995).

[87] D. V. Matyushov and R. Schmid, Calculation of LennardJones energies of molecular fluids, J. Chem. Phys. 104, 8627 (1996).

[88] G. E. Hassan, H. El-Kashef, B. Y. El-Baradie, and M. ElLabban, Measurement of the physical properties of cyclohexane using a laser interferometric technique, Opt. Mater. 5, 327 (1996). 